\title{
Evaluation of DEM, and orthoimage generated from Cartosat-1 with its potential for feature extraction and visualization
}

\author{
Ashutosh Bhardwaj \\ Indian Institute of Remote Sensing (ISRO), Dehradun, India \\ Email adress: \\ ashutosh@iirs.gov.in (A. Bhardwaj)
}

To cite this article:

Ashutosh Bhardwaj. Evaluation of DEM, Orthophoto Generated from Cartosat-1 with its Potential for Feature Extraction and Visualization, American Journal of Remote Sensing. Vol. 1, No. 1, 2013, pp. 1-6. doi: 10.11648/j.ajrs.20130101.11

\begin{abstract}
The present study evaluates the accuracy of Triangulation, Digital Elevation Model (DEM) and orthoimage generated by Cartosat-1 data by using Rational Polynomial Coefficient's (RPC's) provided with the Cartosat-1 satellite stereo (PAN) data. The study area is taken as Jaipur city, Rajasthan. The data acquired over Jaipur, on 18 May' 2005 was analysed. The stereopair comprises of terrain ranging from plain area, agriculture fields, urban area, waterbody and Aravalli hill range. A Differential Global Positioning System (DGPS) survey was conducted to collect ground control points (GCPs) for satellite triangulation, DEM generation, orthoimage generation and subsequent analysis for accuracy assessment. Eighteen GCPs were measured through DGPS survey. Twelve GCPs are used for triangulation and six GCPs were used as check points. The overall RMSE for satellite triangulation is 0.753 pixel. Horizontal accuracy of $2.08 \mathrm{~m}$ and $1.74 \mathrm{~m}$ is achieved in X and $\mathrm{Y}$ direction, respectively. The vertical accuracy of $3.72 \mathrm{~m}$ has been assessed separately also by using independent check point in highly varying terrain.
\end{abstract}

Keywords: Triangulation, DEM, DGPS, RPCs, GCPs

\section{Introduction}

CARTOSAT-1 is the eleventh satellite to be built in the Indian Remote Sensing (IRS) series and was launched on May 5, 2005. This sun synchronous satellite carries two pancharomatic cameras that capture stereoscopic images over a $30 \mathrm{~km}$ swath at $2.5 \mathrm{~m}$ ground resolution enabling the creation of accurate 3D maps. In terms of technology and as a successor of ASTER and SPOT-5 (HRS), Cartosat-1 has proved its potential for High resolution satellite remote sensing having potential of applications in natural resource management and large scale mapping.

The concept of RPC's has been introduced by IKONOS [1]. Many studies have carried out IKONOS stereo data analysis using Rational Function Model (RFM) for evaluating geometric accuracy [2], stereo feature extraction [3], bundle adjustment [4], and mapping [5]. The study of Cartosat-1 stereo data and products reveals DEM accuracy of about 4m [6,7]. Jacobsen, has shown subpixel accuracy for Cartosat-1 stereo scenes. ISPRS-ISRO Cartosat-1 Scientific Assessment Programme (C-SAP) Technical report describes results over test areas Mausanne and Warsaw [8,9]. Nelson and Wilson separately describe various DEM production methods and sources in details [10,11]. Willneff et al. (2008) has compared the geopositioning accuracy achievable with Cartosat-1 imagery via the 3D affine, bias-corrected RPC and generic pushbroom sensor models [12]. YVN Murthy et.al.(2008) from their analysis have stated that the cartosat-1 imagery are capable of producing topographic maps at $1: 25000$, thematic maps at 1:10000 and contour map with contour interval of $10 \mathrm{~m}$ [13]. Singh et al. (2008) has shown results of 5 datasets from Cartosat-1 using RPCs and concluded that RPCs can be provided to the users for photogrammetric processing at their end without compromise on the accuracy [14]. The geometric quality of an ortho-imagery depends on the accuracy of the orientation during Epipolar generation, accuracy of ground control points and on the geometric quality and resolution of the used DEM. All the accuracy of the derived terrain parameters like slope, aspect, drainage, watershed boundary, cut and fill calculations etc, in terms of the position, geometry and orientation, depends on the accuracy of the DEM used to generate or extract these parameters. The determination of exterior orientation parameters is a key element for any kind of imagery from terrestrial, airborne or satellite based sensors. This orientation task traditionally is solved in ana- 
lytical/analog/digital photogrammetry by aerial triangulation with GPS or Kinematic GPS data. RPC's relate object space to image space and vice versa. Even though no GCP's are available, ground co-ordinates can be derived from image co-ordinates using RPC's. The accuracy of the coordinates depends on the source of RPC's. If GCP's are available one can refine the RPC's using polynomial functions.

\section{Objectives}

The objectives of present study were:

- Satellite triangulation of Cartosat-1 data using: 1. RPC's and 2. GCP's.

- To generate the Digital Elevation Model and orthoimage.

- Accuracy assessment of DEM generated using CARTOSAT-1 data.

- Comparision and accuracy assessment of features extracted by using RPC's with that of features extracted by using GCP's collected through DGPS survey.

\section{Methodology}

The methodology adopted for this study is shown in fig.1, with the steps as follows:

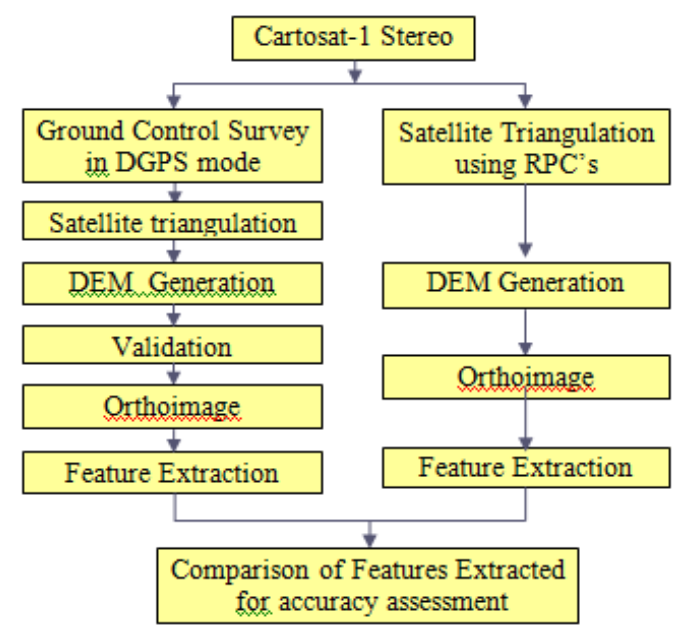

Figure 1. The flowchart of Methodology.

\subsection{Pre-Field Survey Planning}

As a part of pre-field planning, the proposed GCP's locations were identified on the satellite data. The factors considered for the identification of points are clearly identifiable points on both the AFT and FORE images. These points were uniformly placed in such a way that a set of point would be used for generation of DEM and other for validation of DEM.

\subsection{Post-Processing of GPS Data}

The post-processing of the GPS data was carried out using SKI-PRO software package. The GPS survey was conducted in the differential mode using single frequency Leica GPS
500 System. It consists of one pair of instrument, having one base station and one Rover. First the base station is setup and Rovers are setup with unique point id's and stationed for about 1 hour at every GCP location. The system was setup to read the signal for every 20 seconds. There were 18 GCP locations, where Rover was stationed (fig.2).

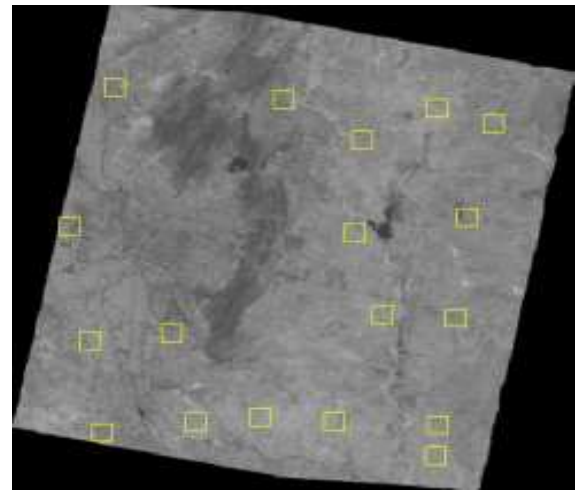

(a)

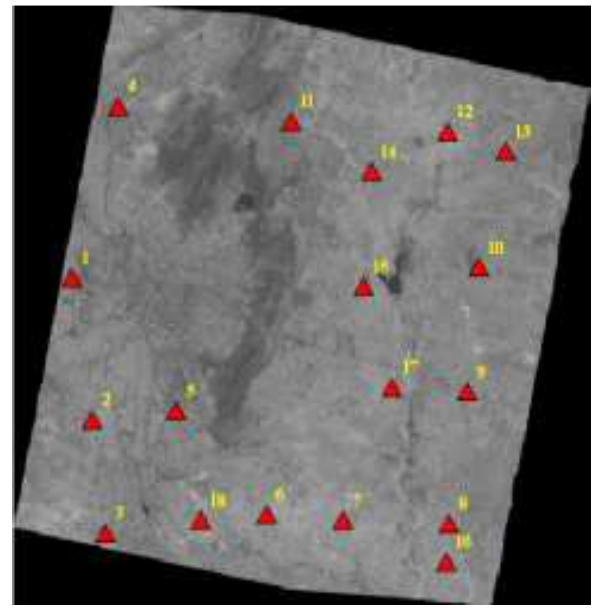

(b)

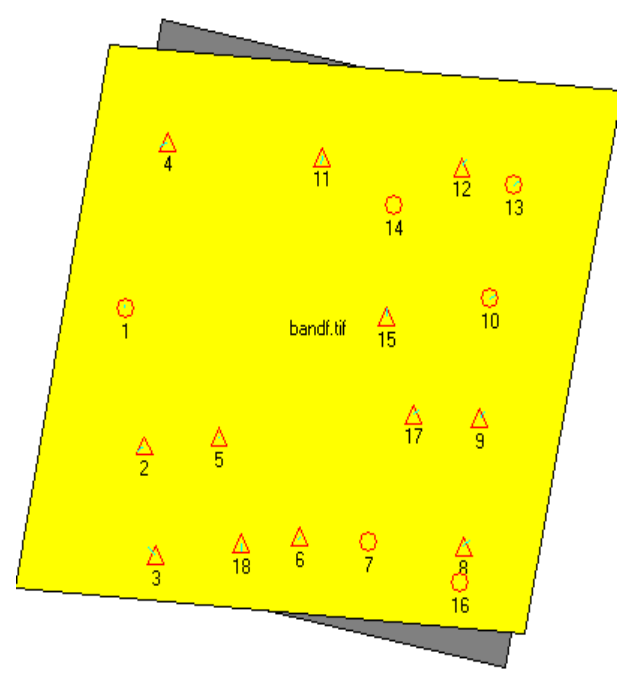

(c)

Fig. 2. Distribution of Ground Control Points: a) Proposed; b) measured; c) control and check points. 


\subsection{Triangulation, DEM and Orthoimage Generation Using RPC's}

Satellite Triangulation was performed using satellite photogrammetric techniques using RPC's available with the Cartosat-1 stereo data in Leica Photogrammetric Suite (LPS) software. Thereafter the DEM and Ortho-image were generated in LPS. In case of CARTOSAT-1, we used the RPC Orthokit. So Interior and Exterior Orientation have been performed automatically because Interior and Exterior Orientation parameters were read from the Orthokit RPC file. DEM generation procedure involves the computation of a model, creation of an epipolar pair and then the extraction of a DEM. The output DEM is shown in fig. 3 and Orthoimage of Cartosat-1 data was generated.

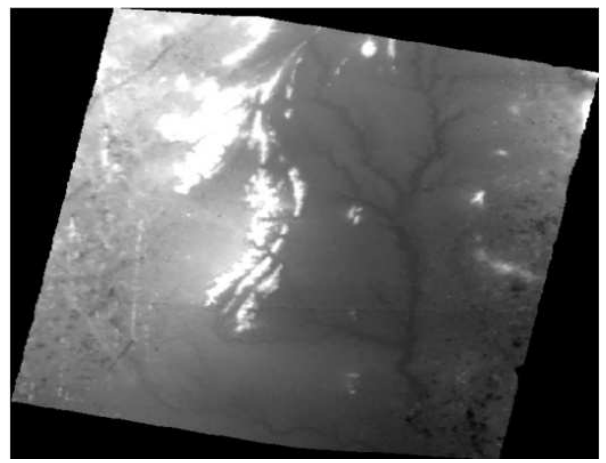

Figure 3. DEM generated by using RPC's (Cartosat-1).

\subsection{Triangulation, DEM and Orthoimage Generation Using GCP's Points}

The Differential GPS survey was carried out to generate ground control points (GCP's) and this dataset was used to perform satellite triangulation and subsequent generation of DEM and orthophoto. The inputs for the generation of DEM are i) Fore and aft image (Stereo pair), ii) Rational Polynomial Coefficients (RPC) files and iii) Co-ordinates of Ground Control Points (GCP's) collected using Differential Global Positioning Survey (DGPS) survey. DEM (fig. 4), and orthoimage (Figure 5) was generated using satellite photogrammetric techniques using Rational Function model. In the present study, these coefficients were updated using GCP's established using DGPS measurements.

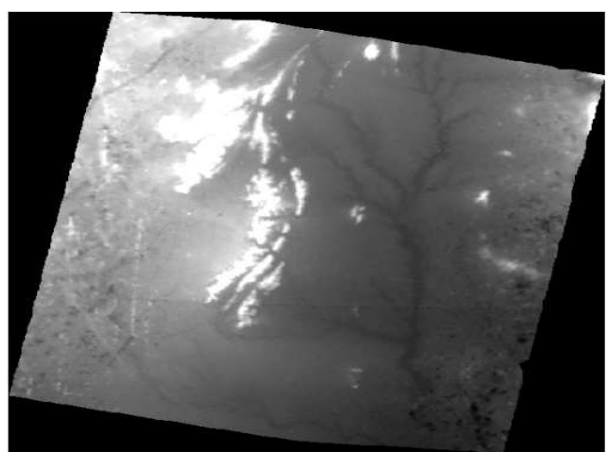

Figure 4. DEM generated using RPC's (Cartosat-1).

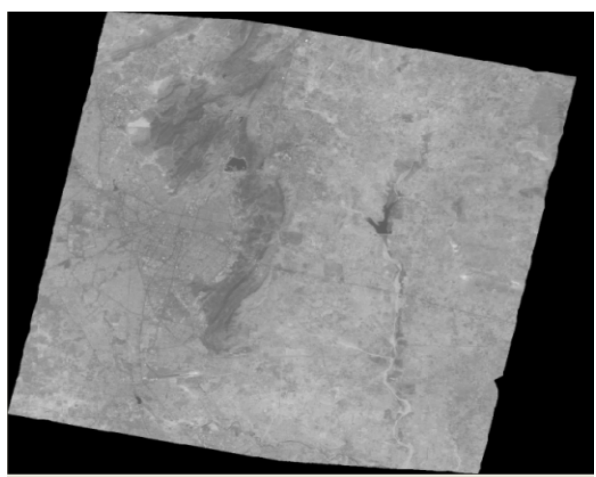

Figure 5. Orthoimage generated using RPC's (Cartosat-1).

In the Triangulation Report, the units for the image coordinates, exterior orientation rotation angles, and GCP's are defined. The positional elements of exterior orientation use the same units as the ground control points. Image coordinate residuals display in the same units as the input image coordinates. In the output image: $\mathrm{x}, \mathrm{y}$ units are in pixels, angle units are in degrees and the ground $\mathrm{X}, \mathrm{Y}, \mathrm{Z}$ units are in meters.

Check points are used to independently verify the quality of the bundle block adjustment. Once the exterior orientation parameters have been solved, the image coordinate values of the check points are used to compute the X, Y, and Z coordinates. The computed coordinates are subtracted from the original input coordinates to compute the check point residuals. Check points serve as the best source for determining the accuracy of the bundle block adjustment.

Once the triangulation solution has converged, the resulting coefficients associated with the exterior orientation parameters display along with their corresponding precision estimates. Once the satellite triangulation solution has converged, ground point values for GCPs, tie points, and check points are computed along with their corresponding accuracy. The ground point information displays as Point ID, Image Number, X (accuracy), Y (accuracy), and Z (accuracy). Various major features were captured and overlaid on orthoimages by digitizing the Orthoimages (Aft) generated using RPC's (fig. 6) and using GCPs for comparision (Fig. 7) respectively.

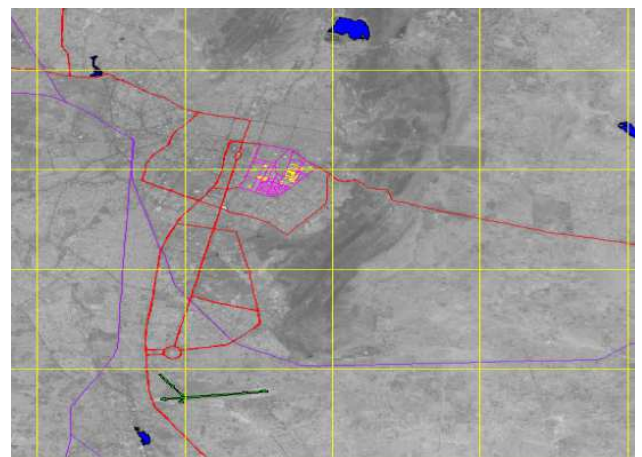

Figure 6. Digitized features overlaid on Orthoimage generated using $R P C$ 's (Cartosat-1). 


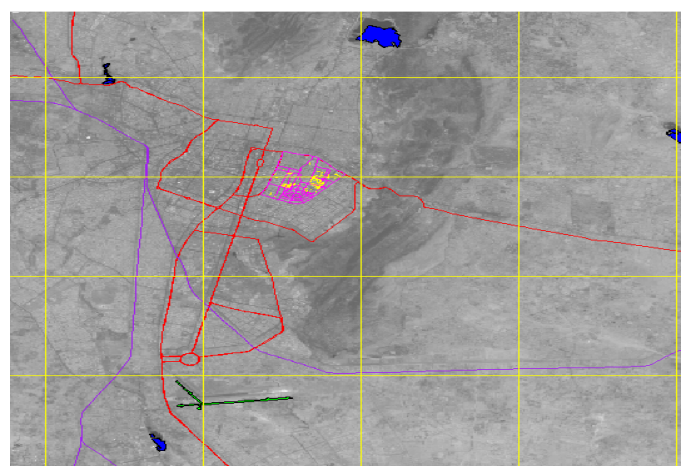

Figure 7. Digitized features overlaid on orthoimage generated using GCP's.

\subsection{Visualization}

For visualization of the information in Cartosat-1 stereo, a fly-through was created in VirtualGIS (ERDAS Imagine). A still (Fig.8) from fly-through shows the Aravalli Hills, waterbody (JalMahal Lake), Jaipur city, and its surroundings very crisply. The visualization reveals an immense potential for planning, execution and monitoring of developmental projects. Along with the recent developments in 3D GIS, this also emphasizes the potential of our present and future high-resolution satellite missions.

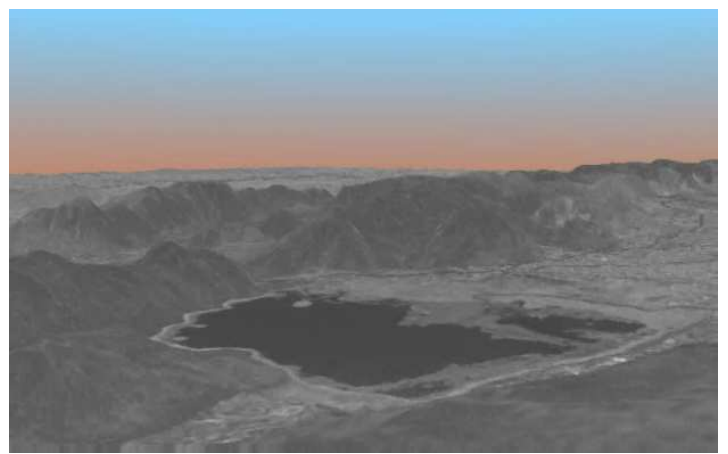

Figure 8. Still from the fly-through generated for visualization.

\section{Results and Analysis}

\subsection{Output Results of DEM and Orthoimage Using RPC's}

The results of satellite triangulation adjustment report for cartosat-1 stereo are as follows for image point residuals having total RMSE of 1.5976:

Image 1: $\mathrm{Ax}=0.0001, \mathrm{Ay}=-0.0003, \mathrm{Mx}=1.1938, \mathrm{My}=$ 0.0095

Image 2: $\mathrm{Ax}=0.0001, \mathrm{Ay}=0.0034, \mathrm{Mx}=1.0532, \mathrm{My}=$ 0.1335

Cartosat-1 DEM was generated (Figure 3) and the corresponding orthoimage was generated (Figure 6).

\subsection{Output Results for Satellite Triangulation Using GCP's}

The overall RMSE for satellite triangulation has come to 0.753 pixel. Horizontal accuracy of $2.08 \mathrm{~m}$ and $1.74 \mathrm{~m}$ is achieved in $\mathrm{X}$ and $\mathrm{Y}$ direction, respectively. The vertical accuracy of $3.72 \mathrm{~m}$ has been assessed separately also by using independent check point in highly varying terrain.

The control point and check point residuals in meters are given in Table 1.

Table 1. Residuals for control and check points

\begin{tabular}{|c|c|c|c|c|}
\hline type & pid & residual_x & residual_y & residual_z \\
\hline gcp & 1 & -3.66478601 & 2.74769640 & -4.09382774 \\
\hline gcp & 2 & -4.25076435 & -4.68064508 & 2.89869165 \\
\hline gcp & 3 & -4.59698494 & 2.90112146 & -2.53927122 \\
\hline gcp & 4 & -0.08518215 & 1.49785460 & 4.11053237 \\
\hline gcp & 5 & -0.82303960 & 1.82606078 & 2.37010133 \\
\hline gcp & 6 & 4.72150303 & -4.70173737 & 3.24819128 \\
\hline gcp & 7 & 2.89822447 & -3.56503672 & -1.74091765 \\
\hline gcp & 8 & -0.61604752 & 4.40789786 & 3.51279305 \\
\hline gcp & 9 & 3.16528696 & -3.77766758 & 1.96458842 \\
\hline gcp & 10 & -0.13446086 & -4.37412795 & -4.76341164 \\
\hline gcp & 11 & 4.73182900 & -2.20874822 & -2.18919774 \\
\hline gcp & 12 & 0.21881739 & 4.95610532 & 4.88599617 \\
\hline chk & 1 & -1.03521619 & -2.17741409 & 3.54399092 \\
\hline chk & 2 & 0.15297512 & -0.15388558 & 4.11536963 \\
\hline chk & 3 & 3.59418935 & -2.19791556 & -3.80890849 \\
\hline chk & 4 & 3.46698428 & -2.95880900 & -4.62874990 \\
\hline chk & 5 & 0.15233707 & -0.15370054 & 3.47978221 \\
\hline chk & 6 & 0.00413465 & -0.02497913 & -2.40369539 \\
\hline
\end{tabular}

Image point residuals:

$$
\begin{aligned}
& \mathrm{Ax}=0.0030, \mathrm{Ay}=-0.0346, \mathrm{Mx}=1.1842, \mathrm{My}=0.4345 \\
& \mathrm{Ax}=-0.0510, \mathrm{Ay}=-0.0455, \mathrm{Mx}=1.2890, \mathrm{My}=0.5247
\end{aligned}
$$

The Ax, Ay, and Az values reflect the average residual values for the $\mathrm{X}, \mathrm{Y}$, and $\mathrm{Z}$ control point coordinates, respectively. The $\mathrm{Mx}, \mathrm{My}$, and $\mathrm{Mz}$ values reflect the root mean square errors (standard deviation) of all $\mathrm{X}$, Y, and $\mathrm{Z}$, respectively. The summary of RMSE of GCP's is given in Table 2. Image accuracy for control and check points for each scene are given in Table 3. 
Table 2. Image accuracy for control and check points for each scene.

\begin{tabular}{lllll}
\hline \multicolumn{2}{c}{ pid type } & image id 1 & & Image id 2 \\
& residual_x & residual_y & residual_x & residual_y \\
\hline 1 & -0.4872 & 0.6460 & 2.8288 & 2.0663 \\
2 & 0.7304 & -2.0434 & 2.3136 & -2.5394 \\
3 & 0.5086 & 0.6786 & 2.9218 & 1.7218 \\
4 & 0.0820 & 0.7009 & 0.9284 & -0.3502 \\
5 & 1.0408 & 0.7069 & 0.3508 & 0.1413 \\
6 & -0.6972 & -1.3350 & -3.1431 & -2.5822 \\
7 & -0.5345 & -1.1979 & -2.4937 & -1.0531 \\
8 & 0.5321 & 1.7569 & 1.2231 & 1.0005 \\
9 & -0.5009 & -1.1420 & -2.1838 & -1.9343 \\
10 & -0.4813 & -1.8422 & -0.8791 & -0.7188 \\
11 & -1.3086 & -0.5406 & -3.0489 & -0.3629 \\
12 & 0.4338 & 2.0780 & 1.0042 & 0.9313 \\
\hline
\end{tabular}

Check Point Residuals

\begin{tabular}{lllll}
\hline 1 & -1.0930 & -0.7983 & 2.0392 & -1.4760 \\
2 & 1.5136 & 0.0713 & -0.8275 & -1.0938 \\
3 & -0.5066 & -0.6833 & -3.1490 & -0.0894 \\
4 & -0.7977 & -1.0103 & -3.0329 & -0.1916 \\
5 & 0.4449 & 0.0595 & 0.0258 & -0.8427 \\
6 & 1.0737 & -0.0963 & -1.3110 & 0.3725 \\
\hline
\end{tabular}

Table 3. Summary RMSE for GCPs and CHKs (number of observations in parenthesis).

\begin{tabular}{lll}
\hline & Control & Check \\
\hline Ground X: & $3.120678(12)$ & $2.083925(6)$ \\
Ground Y: & $3.657680(12)$ & $1.749973(6)$ \\
Ground Z: & $3.357331(12)$ & $3.726389(6)$ \\
Image X & $1.605638(24)$ & $1.617778(12)$ \\
Image Y & $1.436384(24)$ & $0.730941(12)$ \\
\hline
\end{tabular}

\subsection{Accuracy Assessment of Digital Elevation Model}

To validate the accuracies of DEM generated through Cartosat-1 data the check points measured by DGPS surveying techniques were used. Accuracy assessment was done by using 6 check points. The RMSE in elevation was $3.72 \mathrm{~m}$, i.e. about 1.48 pixel. One point in between hills is also used for validation besides 6 check points. This concludes that even in a location surrounded by the hills (Jaipur), the automatically generated DEM using RPC gives accuracy within 2 pixels. It was observed that while using only RPC's information for Cartosat-1 stereo data, the shift in height was in the range 230 to $248 \mathrm{~m}$ (Table 2) which is actually attributed to shift in the planimetric position of the points. By using twelve GCP's as control points along with six GCP's as check points for Cartosat -1 stereo data, the shift in height was highly improved and resulted in the range 0.05 to $9.1 \mathrm{~m}$ for difference in height (Table 4).

Table 4. Comparision of height from DEM generated using $R P C C^{\prime} S / G C P$ 's.

\begin{tabular}{|c|c|c|c|c|c|}
\hline Point ID no. & $\begin{array}{l}\text { Z Height } \\
\text { (from DEM) } \\
\text { Meters WGS84 }\end{array}$ & $\begin{array}{l}\text { Z' Height } \\
\text { (from GPS) } \\
\text { Meters WGS84 }\end{array}$ & $\begin{array}{l}\text { Z Height Diff. } \\
\left(Z^{\prime}-Z^{\prime}\right) \\
\text { Meters }\end{array}$ & $\begin{array}{l}\text { " Height } \\
\text { (from DEM Generated } \\
\text { using RPC) } \\
\text { Meters } \\
\end{array}$ & $\begin{array}{l}\text { Height Diff. } \\
\left(Z^{\prime \prime}-Z^{\prime}\right) \\
\text { Meters }\end{array}$ \\
\hline \multicolumn{6}{|c|}{ Points used as GCP's Control Point } \\
\hline 1 & 366.21 & 358.19 & 8.02 & 594.78 & 236.59 \\
\hline 2 & 327.58 & 324.16 & 3.42 & 566.51 & 242.35 \\
\hline 3 & 420.18 & 423.65 & -3.47 & 654.05 & 230.39 \\
\hline 4 & 366.54 & 357.39 & 9.14 & 604.61 & 247.22 \\
\hline 5 & 313.73 & 311.87 & 1.85 & 554.77 & 242.90 \\
\hline 6 & 285.52 & 290.72 & -5.20 & 525.83 & 235.10 \\
\hline 7 & 298.84 & 301.99 & -3.14 & 539.52 & 237.53 \\
\hline 8 & 343.84 & 342.02 & 1.81 & 589.40 & 247.37 \\
\hline 9 & 325.62 & 332.62 & -7.00 & 568.55 & 235.93 \\
\hline 10 & 310.50 & 316.97 & -6.47 & 550.27 & 233.29 \\
\hline 11 & 297.66 & 302.97 & -5.31 & 535.20 & 232.22 \\
\hline 12 & 320.24 & 316.06 & 4.18 & 560.32 & 244.26 \\
\hline \multicolumn{6}{|c|}{ GCP's which are used as Check Point } \\
\hline 1 & 374.45 & 375.08 & -0.62 & 610.22 & 235.14 \\
\hline 2 & 300.22 & 300.17 & 0.04 & 540.51 & 240.33 \\
\hline 3 & 327.63 & 327.29 & 0.33 & 561.50 & 234.20 \\
\hline 4 & 351.35 & 353.28 & -1.93 & 593.42 & 240.13 \\
\hline 5 & 321.56 & 326.92 & -5.36 & 561.11 & 234.18 \\
\hline 6 & 278.92 & 286.53 & -7.61 & 517.04 & 230.51 \\
\hline
\end{tabular}

\subsection{Accuracy Assessment of Sample Digital Maps}

The orthoimage's were generated using RPC's and than using GCP's. The features extracted from orthoimages prepared by RPCs alone and by using GCPs were overlaid for comparision. The shift in the features digitized was calculated for positioning. The improvement in locational positioning after using GCP's in X and Y were in range of 74.25 to $85.75 \mathrm{~m}$ and 279.25 to $294.75 \mathrm{~m}$ respectively (table 4.1 ). 


\section{Conclusion}

The DEM generated from Cartosat -1 stereo data, through automatic mode is accurate to better than two pixels to 2.5 pixels depending on the topography and it can be useful for topographic analysis. It was also observed that accuracy of DEM generated from Cartosat-1 Stereo data could be improved with using good distribution of GCP's, although less number of GCP's giving a peripheral control on four corners and one at the centre of scene can also be used. The geometric accuracy and information potential of Orthoimages and DEM provided by the Cartosat-1 Mission can be exploited for topographic mapping at 1:25,000 to $1: 10,000$ scale with varying and appropriate number of layers and details. It also has a good potential for terrain visualization.

\section{Acknowledgements}

The author is thankful to Dr. V. K. Dadhwal, Director, National Remote Sensing Centre, Hyderabad for his guidance and help during the execution of the presented work.

\section{References}

[1] Dial, G. (2000). IKONOS satellite mapping accuracy. ASPRS 2000 Proceedings, Washington DC, 22-26 May 2000 .

[2] Grodecki, J and G. Dial (2001). IKONOS geometric accuracy. Proceedings of Joint Workshop of ISPRS WorkingGroups I/2, I/5 and IV/7 on High Resolution Mapping from Space 2001, University of Hanover, Hanover, Germany, Sept 19-21.

[3] Grodecki, J. (2001). IKONOS stereo feature extraction - RPC approach. ASPRS 2001 Proceedings, St. Louis, MO, 23-27 April 2001.

[4] Grodecki, J. and G. Dial (2003). Block adjustment of high-resolution satellite images described by rational polynomials. Photogrammetric Engineering and Remote Sensing, 69(1):59-68.

[5] Tao, C.V., Hu,Y. and Jiang, W.(2004). Photogrammetric exploitation of IKONOS Imagery for Mapping Application. International Journal of Remote Sensing, 25(14):2833-2853.

[6] Krishnaswamy, M. and S. Kalyanaraman (2004). "Indian Remote Sensing Satellite Cartosat-1: Technical features and data products."

[7] Nadeem, Ahmed., Anjum, Mahtab., Ritesh Agrawal., R, Jayaprasad., S.K., Pathan., Ajai, D.K., SINGH., Anda.K. SINGH,. Extraction and Validation of Cartosat-1 DEM, Journal of the Indian Society of Remote Sensing, Vol. 35, No. 2, 2007.

[8] Jacobsen K., 2006: ISPRS-ISRO Cartosat-1 Scientific Assessment Programme (C-SAP) Technical report - test areas Mausanne and Warsaw, ISPRS Com IV, Goa 2006, IAPRS Vol. 36 Part 4, pp. 1052-1056.

[9] Jacobsen, K., Crespi, M., Fratarcangeli, F., Giannone, F, 2008.: DEM generation with Cartosat-1stereo imagery EARSel, Workshop Remote Sensing - New Challenges of High Resolution, Bochum 2008.

[10] Nelson, A., Reuter, H.I., Gessler, P., 2009. DEM production methods and sources. In: Hengl, T., Reuter, H.I. (Eds.), Geomorphometry: Concepts, Software, and Applications. Elsevier, Amsterdam, pp. 65-85.

[11] John P. Wilson, Digital terrain modeling, Geomorphology, Volume 137, Issue 1, 15 January 2012, Pages 107-121.

[12] Willneff, J., Weser, T., Rottensteiner, F., and C. S. Fraser (2008). "Precise Georeferencing of Cartosat Imagery via different orientation models", The International Archives of the Photogrammetry, Remote Sensing and Spatial Information Sciences. Vol. XXXVII. Part B1. Beijing 2008.

[13] Krishna Muthry, Y.V.N., Srinivasa Rao, S., Prakasa Rao, D.S., Jayaraman V. Analysis of DEM generated using Cartosat-I stereo data over Mausanne les Alphilles - Cartosat scientific appraisal programme (CSAP TS-5). ISPRS 2008, 37, 13431348.

[14] Singh, S. K., Naidu, D. S., Srinivasan, T.P., Krishna, B. G., and P K Srivastava (2008). "Rational polynomial modeling for Cartosat-1 data", The International Archives of the Photogrammetry, Remote Sensing and Spatial Information Sciences. Vol. XXXVII. Part B1. Beijing. 